\title{
Decay properties of unstable Tonks-Girardeau gases from a split trap
}

\author{
Przemysław Kościk \\ Department of Computer Sciences, State Higher Vocational School in Tarnów, ul. Mickiewicza 8, PL-33100 Tarnów, Poland*
}

\begin{abstract}
We study the decay properties of Tonks-Girardeau gases escaping from a double-well potential. Initially, the gases are constrained between two infinite $\delta$ barriers with an on-center $\delta$-split potential. The strength of one of the obstacles is suddenly reduced, and the particles start to tunnel to the open space. Using the resonance expansion method, we derive the single-term approximate expression for the $N$-particle survival probability and demonstrate its effectiveness in both the exponential and nonexponential regimes. We also predict a parity effect and provide physical insights into its nature at different stages of the time evolution. We conclude that only the initial phase of the decay of the many-particle state may comply with an exponential law. The decay properties are dramatically affected by the presence of the split barrier. Our results reveal the overall decay mechanism of unstable Tonks-Girardeau gases from single and double quantum wells.
\end{abstract}

\section{INTRODUCTION}

Since the birth of quantum physics, there has been much interest in the properties of particles tunneling from an open well trap [1-4]. Many research studies have been conducted so far in this context, and various models have been considered in the literature [5-10]. In particular, the so-called Winter model [11], consisting of an infinite wall and a $\delta$-function potential, has attracted significant attention [12-17]. A good feature of the Winter's model is that its energy eigenfunctions can be found in closed analytical forms, enabling us to gain insights into the properties of the decaying particles easily. With this concrete example, Winter [11] showed that the decay process exhibits deviations from the exponential law, and its long-time evolution follows a power law. The nonexponential decay has been observed in a variety of experiments [18-20]. New advances in experimental techniques have provided opportunities to study tunneling phenomena in a controllable way [20-22], thereby accelerating research activity towards a better understanding of the properties of unstable many-body states. For instance, the recent studies include systems of two identical noninteracting particles [23], ultracold-atom systems [24-31], two-particle systems with Coulomb interactions [32], and a model system consisting of a core nucleus and two valence protons [33]. It is worth mentioning that the complex scaling method [34] also provides a tool for studying the resonance states of manyparticle systems [35-38]. However, few attempts have been made to increase our understanding of the decay properties of systems with more than two particles.

One of the essential results regarding the onedimensional (1D) systems is the famous Bose-Fermi (BF) mapping [39]. In the most straightforward case, the BF mapping relates the wave function of bosons with infinitely strong $\delta$ repulsions (Tonks-Girardeau gas) to a free-fermion gas. The $\mathrm{BF}$ mapping also holds for the time-dependent case [40]. As a result, the theoretical

\footnotetext{
*p_koscik@pwsztar.edu.pl
}

investigations of unstable Tonks-Girardeau (TG) gases can be considerably simplified, which offers unique opportunities to gain physical insights into the tunneling process at the few-body level. Some researchers have taken action to explore the decay properties of TG gases $[7,15,16]$, where one of the relevant results concerns the long-time decay [16].

This work aims to carry out a comprehensive investigation of the properties of unstable TG gases escaping from an open double-well potential. Our interest in such systems is primarily motivated by the implementation of double-well structures in laboratories and theoretical works in this area [31, 41-48]. A simple candidate by which to simulate a double-well structure is Winter's model with an additional $\delta$-split barrier at the center of the trap.

We examine the typical scenario of the tunneling process; namely, the $N$ hardcore bosons are initially confined in the hard-wall split trap. At some time, one of the barriers is lowered, and the initial state starts to decay in time. Within the framework of the resonance expansion approach [10], we identify the tunneling mechanisms in different time regions by providing a closed-form approximate expression for the $N$-particle survival probability. Our results go beyond the long-time decay and, in this sense, they extend the results in Ref. [16]. We also address the question of how the decay properties are influenced by the $\delta$-split barrier when changing its strength and the number of particles, $N$.

The remainder of this study is as follows. In Sec. II, we present the model and theoretical tools used to probe its decay properties. Section III is devoted to the features of decaying one- and many-particle states. Some concluding remarks are found in Sec. IV.

\section{MODEL}

The following Hamiltonian describes the 1D system of $N$ indistinguishable bosons interacting via a contact potential: 


$$
\hat{\mathcal{H}}=\sum_{i=1}^{N} \hat{h}(i)+g \sum_{j<k} \delta\left(x_{j}-x_{k}\right),
$$

where $\hat{h}$ is the single-particle Hamiltonian and $g$ is the strength of the interaction [49]. For the present system, the Hamiltonian $\hat{h}$ takes the following form:

$$
\hat{h}=-\frac{\hbar^{2}}{2 m} \frac{\mathrm{d}^{2}}{\mathrm{~d} x^{2}}+\mathcal{V}(x)+\alpha \delta(x)+\eta \delta(x-L),
$$

with $\alpha \geq 0$ and $\eta \geq 0$, where $\mathcal{V}(x)$ represents a hardwall trap that is infinite in the region $x<-L$ and zero elsewhere, and $2 L$ is the width of the trapping potential. With the emergence of new technologies, such a 1D box split trap can be experimentally fabricated by laser trapping techniques. In the TG limit $(g \rightarrow \infty)$ that we are interested in, the bosonic wave function is related via $\mathrm{BF}$ mapping [39] to the corresponding wave function of spineless free fermions as follows:

$$
\Psi\left(x_{1}, x_{2}, \ldots, x_{N}\right)=\hat{\Upsilon} \Psi_{F}\left(x_{1}, x_{2}, \ldots, x_{N}\right),
$$

where $\hat{\Upsilon}$ is the mapping function $\hat{\Upsilon}=\Pi_{k<l} \operatorname{sgn}\left(x_{k}-x_{l}\right)$ that ensures the bosonic symmetry ( $\operatorname{sgn}$ is the sign function). We choose the initial state $(t<0)$ as the ground state of $N$ hardcore bosons in a hard-wall split trap. At time $t=0$, the barrier strength at $x=L$ is instantaneously reduced to a finite value of $\eta$, and the initial wave function starts to evolve with time [40]:

$$
\begin{array}{r}
\Psi\left(x_{1}, x_{2}, \ldots, x_{N} ; t\right)= \\
=\Pi_{k<l} \operatorname{sgn}\left[x_{k}-x_{l}\right] \frac{1}{\sqrt{N !}} \operatorname{det}_{i=1, j=1}^{N}\left[\phi_{i}\left(x_{j}, t\right)\right],
\end{array}
$$

where det symbolizes the determinant and $\phi_{n}(x, 0)$ is the $n$th eigenstate of the single-particle Hamiltonian (2) in the hard-wall limit $(\eta \rightarrow \infty)$. The evolution process of each single-particle orbital is governed by the Schrödinger equation:

$$
i \hbar \frac{\partial \phi_{k}(x, t)}{\partial t}=\hat{\mathcal{H}} \phi_{k}(x, t) .
$$

In the following, we take $L=\hbar=m=1$; i.e., the spatial and time coordinates are measured in units of $L$ and $m L^{2} / \hbar$, respectively, and energies in units of $\hbar^{2} /\left(m L^{2}\right)$. The model we consider has a convenient feature where both the continuum wave functions $(\eta<\infty)$ and the eigenfunctions of the hard-wall split trap can be found in closed analytical forms (see Appendixes VA and VB). The solutions to Eq. (5) maybe written in terms of the continuum wave functions as follows:

$$
\phi_{k}(x, t)=\int_{0}^{\infty} C_{k}(p) \psi_{p}(x) e^{-\frac{i t p^{2}}{2}} \mathrm{~d} p
$$

where

$$
C_{k}(p)=\int_{-1}^{1} \phi_{k}(x, 0) \psi_{p}(x) \mathrm{d} x
$$

The integrals in Eq. (7) can be performed explicitly (Appendix VC). In contrast, those in Eq. (6) need numerical integration.

For simplicity, we analyze the decay properties in terms of the survival probability, also known as quantum fidelity, as follows:

$$
S(t)=|\langle\Psi(0) \mid \Psi(t)\rangle|^{2},
$$

which measures how the time-evolved state differs from the initial state. For the TG gases, the $N$-particle survival probability can be simplified to the following form [16]:

$$
S^{(N)}(t)=\left|\operatorname{det}_{k, l=1}^{N}\left[\mathcal{A}_{k l}(t)\right]\right|^{2},
$$

where $\mathcal{A}_{k l}(t)=\left\langle\phi_{k}(0) \mid \phi_{l}(t)\right\rangle$. With the use of integral representation in Eq. (6), $\mathcal{A}_{k l}(t)$ can be expressed by the following 1D integrals:

$$
\mathcal{A}_{k l}(t)=\int_{0}^{\infty} C_{k}^{*}(p) C_{l}(p) e^{-\frac{i t p^{2}}{2}} \mathrm{~d} p,
$$

which makes calculating the survival probability less complicated than the nonescape probability, both in the framework of the resonance expansion method [10] and numerically. The integrals in Eq. (6) are highly oscillatory; thus, their accurate estimation is a very tedious numerical task. One has to use the resonance expansion method to obtain insights into the decay properties in the long-time regime, especially when $N$ is large.

However, the survival and nonescape probabilities have their resonance expansions in the same form [10] (only the expansion coefficients are different). As a result, each characteristic behavior of the former (exponential decay, long-time decay, etc.) is usually reflected by the latter, and vice versa $[7,8,23]$. Specifically, such behaviors of both quantities can be described by suitable single-term approximations extracted from their resonance expansions, which are proportional to each other. This was adequately demonstrated in Ref. [23] for two noninteracting particles in entangled symmetric or antisymmetric initial states. In other words, at least in the cases of TG gases, which can be described with the resonance expansion approach, the conclusions inferred from the behaviors of the survival and nonescape probabilities are consistent in the main points.

Within the resonance expansion approach, which is based on Cauchy's residue theorem, Eq. (10) can be rewritten in the form

$$
\mathcal{A}_{k l}(t)=\sum_{r} M_{r}^{(k, l)} e^{-i t p_{r}^{2} / 2}+M^{(k, l)}(t),
$$

where $p_{r}$ are the so-called proper poles, i.e., the roots of the denominator in Eq. (10) on the fourth quadrant of the complex $p$ plane, and $M^{(k, l)}(t)$ is the integral contribution along a path. The exponential terms in Eq. (11) may be rewritten as $e^{-i t p_{r}^{2} / 2}=e^{-\Gamma_{r} t / 2+i t \xi_{r}}$, where $\Gamma_{r}=-\operatorname{Im}\left[p_{r}^{2}\right]$ and $\xi_{r}=\left(\operatorname{Im}\left[p_{r}\right]^{2}-\operatorname{Re}\left[p_{r}\right]^{2}\right) / 2$ can be 

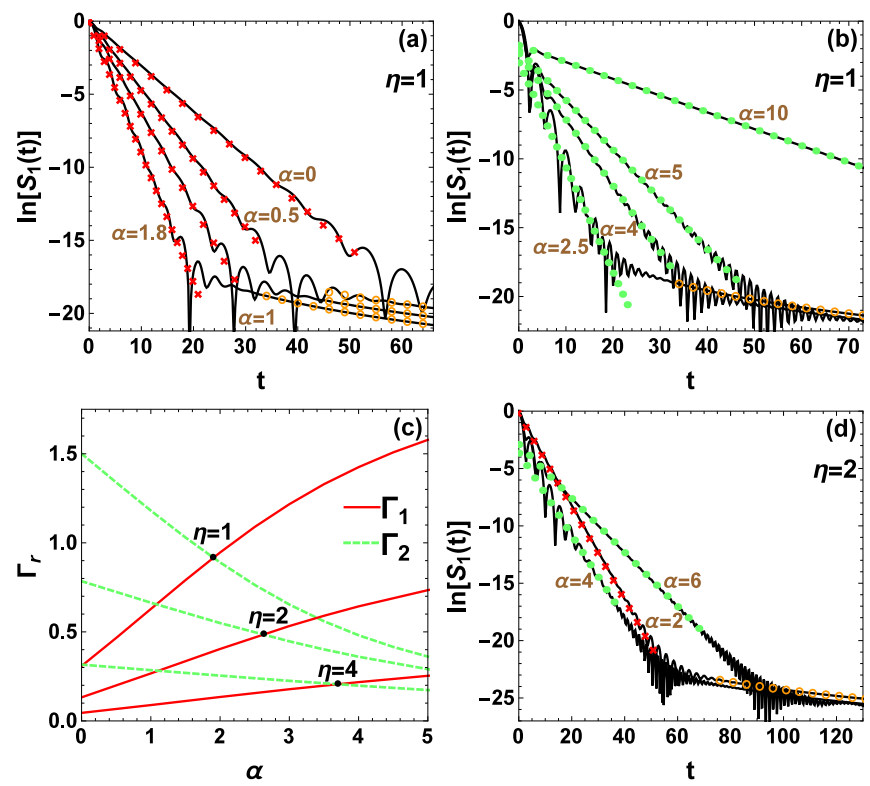

FIG. 1. Results for the lowest-energy initial state, Eq. (29). $(\boldsymbol{a}, \boldsymbol{b}, \boldsymbol{d})$ Results for $S_{1}(t)$ obtained for different values of $\alpha$ and $\eta$ as functions of time $t$. The continuous black lines represent the exact numerical results. The red crosses and green dots mark the results obtained from the one-pole approximations, $S_{1}(t) \approx\left|M_{r}^{(1)}\right|^{2} e^{-\Gamma_{r} t}$, for $r=1$ and for $r=2$, respectively. The orange circles represent the results obtained from the asymptotic formula of Eq. (13). (c) Dependencies of $\Gamma_{1}$ and $\Gamma_{2}$ as functions of $\alpha$ for $\eta=1,2$ and $\eta=4$. The time is given in $m L^{2} / \hbar$, strengths of the barriers in $\hbar^{2} /(m L)$ and decay rates in $\hbar /\left(m L^{2}\right)$.

viewed as the decay rates and resonance energies, respectively. The positions of the proper poles depend only on the system parameter values, and the same applies to $\Gamma_{r}$ and $\xi_{r}$. The nonexponential component $M^{(k, l)}(t)$ can be expanded into a series of inverse powers of $t$, $\sim t^{-n-1 / 2}[10,23]$. Since the integrand in Eq. (10) is given in analytical form, this can be achieved explicitly (at least term by term) [23]. In practice, it suffices to take into account in Eq. (11) only a few terms from the poles $p_{r}$ and inverse power contributions to achieve satisfactory approximations, except for short times [50]. For details regarding the resonance expansion approach, see Refs. [10, 16, 23]. From this point onwards, we assume that $\Gamma_{r}$ are ordered according to the resonance spectra: $\xi_{1}<\xi_{2}<\ldots<\xi_{r}<$. For individual states, we denote $M_{r}^{(k)}=M_{r}^{(k, k)}$ and $\mathcal{A}_{k}(t)=\mathcal{A}_{k k}(t)$, and $S_{k}(t)=\left|\mathcal{A}_{k k}(t)\right|^{2}$.

\section{RESULTS}

\section{A. Single-particle case}

First, we shed light on the effect of a split barrier on the decay properties of one particle that is initially in the
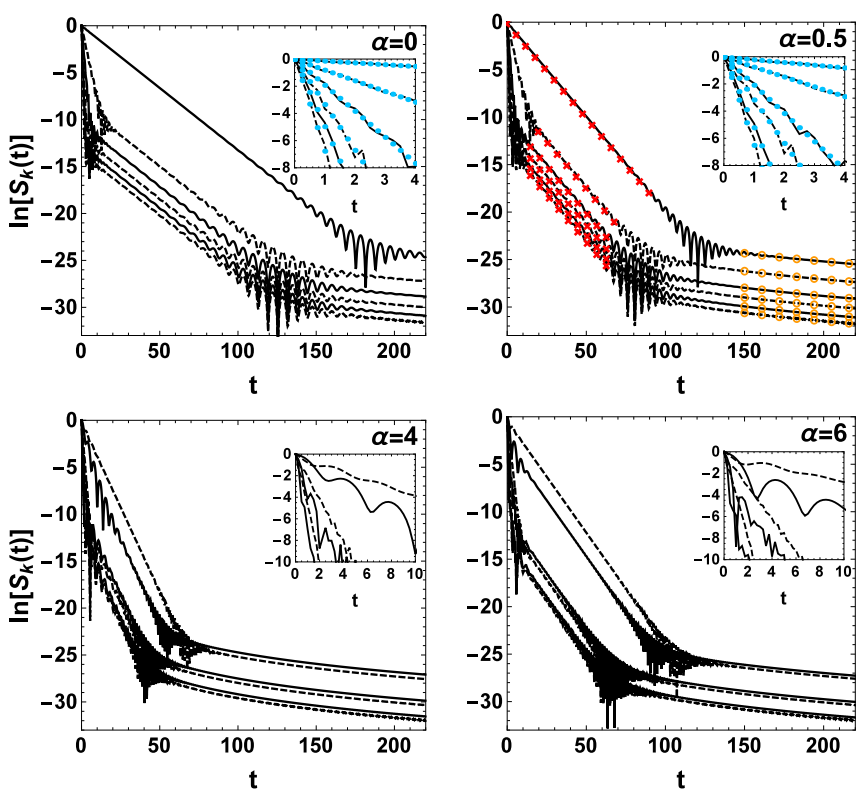

FIG. 2. Survival probabilities of six lowest-energy initial states obtained for some transparent values of $\alpha$ at $\eta=2$. The continuous and dashed black lines represent the exact results for the even- and odd-parity initial states, respectively. In the plot of $\alpha=0.5$, the red crosses and orange circles mark the results obtained from $S_{k}(t) \approx\left|M_{1}^{(k)}\right|^{2} e^{-\Gamma_{1} t}$ and Eqs. (13) and (14), respectively. The blue dots in the insets show the results obtained from $S_{k}(t) \approx e^{-\Gamma_{k} t}(\alpha=0$ and $\alpha=0.5)$. The time and strength of the barriers are in $m L^{2} / \hbar$ and $\hbar^{2} /(m L)$, respectively.

lowest energy state, Eq. (29). Our results are shown in Fig. 1, where the exact results for $S_{1}(t)$ are obtained by employing the numerical estimation of the integrals in Eq. (10). An exponential decay begins in the early period. As $\alpha$ increases, the slope of $\ln S_{1}(t)$ initially decreases, and when $\alpha$ exceeds some critical value, it starts to exhibit the opposite behavior. To gain deeper insights into this effect, we resort to the resonance expansion of the survival amplitude in Eq. (11). Figure 1(c) shows the results obtained for the two lowest decay rates $\Gamma_{r}$ as functions of $\alpha$. The curves intersect at a certain point $\alpha_{c r}$, which suggests that the following single-pole approximation holds:

$$
S_{1}(t) \approx\left|M_{r}^{(1)}\right|^{2} e^{-\Gamma_{r} t},
$$

where $r=1$ for $\alpha<\alpha_{c r}$ and $r=2$ for $\alpha>\alpha_{c r}$. Its applicability is verified in Figs. $1(\boldsymbol{a}), 1(\boldsymbol{b})$, and $1(\boldsymbol{d})$. For values of $\alpha$ differing considerably from $\alpha_{c r}$, it reasonably reproduces the corresponding exact results. For $\alpha \approx \alpha_{c r}$, the resonance terms with $r=1,2$ in Eq. (11) contribute substantially, as reflected by the strong oscillations in the behavior of $S_{1}(t)$. The appearance of the transition point $\alpha_{c r}$ can be attributed to the fact that, at large enough values of $\alpha$, the average energy of the lowest decaying state approaches that of the first excited state. As a consequence, the decay energy spectrum spreads more 
around the second resonance energy $\xi_{2}$, which corresponds to the decaying fragment with $\Gamma_{2}$. Strictly in the limit $\alpha \rightarrow \infty$, the adjacent even- and odd-parity initial states [Eqs. (29) and (30), respectively] become degenerate, which means that their decays proceed in the same way.

Figure 2 shows the behaviors of the survival probabilities for the six lowest-energy initial states. It depicts how the results obtained for the even-parity states converge to those obtained for the odd ones as $\alpha$ is increased. In the weak-split-barrier regime, all the states under consideration undergo exponential decay in short times. The profiles of $S_{k}(t)$ can be reproduced well by $S_{k}(t) \approx e^{-\Gamma_{k} t}$ [7], which is demonstrated in the insets of Fig. 2 (the cases $\alpha=0$ and $\alpha=0.5$ ). As time progresses, the contributions from the poles gradually fade. As a result, after a sufficiently long period, the decays of the excited states proceed with the same decay constant as that of the lowest-energy state [15]. Then, the following approximation applies: $S_{k}(t) \approx\left|M_{r}^{(k)}\right|^{2} e^{-\Gamma_{r} t}$, which is confirmed with an example with $\alpha=0.5(r=1)$ in Fig. 2. Eventually, the decay of $S_{k}(t)$ follows a long-time inverse power law [10], which is derived in analytic form for both even- and odd-parity initial states:

$S_{k}^{(+)} \sim \frac{32 \mathrm{~A}_{k}^{4}\left\{\alpha^{2} p_{k} \cos \left(p_{k}\right)-\left[\alpha^{2}+(\alpha+1) p_{k}^{2}\right] \sin \left(p_{k}\right)\right\}^{4}}{\pi \alpha^{4} p_{k}^{8}[4(\alpha+1) \eta+2 \alpha+1]^{4} t^{3}}$

and

$$
S_{k}^{(-)} \sim \frac{32(\alpha+1)^{4}}{\pi^{5} k^{4}[4(\alpha+1) \eta+2 \alpha+1]^{4} t^{3}},
$$

respectively. As may be seen (Figs. 1 and 2), the results from Eqs. (13) and (14) accurately agree with the numerical ones, which confirms their accuracy and correctness of our calculations.

A feature worth stressing here is that in the regimes of values of $\alpha$ smaller (larger) than $\alpha_{c r}$, the transition time to the long-time $t^{-3}$ regime decreases (increases) with an increase in $\alpha$. It can thus be concluded that by appropriately choosing the strength of the splitting barrier, one can accelerate or slow down the decay process, keeping the strength of the trapping potential fixed at the same time.

\section{B. N-particle case}

Now, we come to the main point, where we explore the decay properties of the TG gases [39]. Below, we restrict our investigation to cases with sufficiently low split barriers without losing much generality. Our results for the $N$-particle survival probability $S^{(N)}(t)$ are summarized in Fig. 3, where, for the sake of clarity, we only depict the results for $\alpha=0$ and $\alpha=2$. Figure 3(a) shows the results obtained using the resonance expansion approach. In contrast, the results of Fig. $3(\boldsymbol{b})$ highlight the
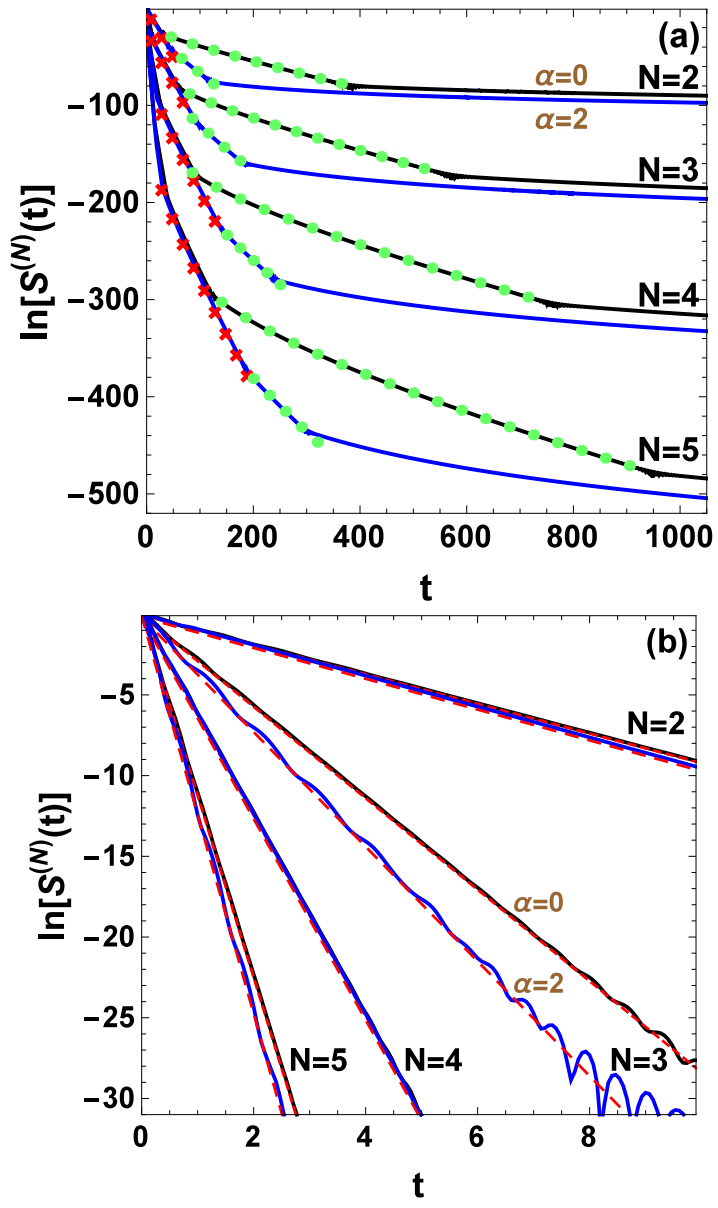

FIG. 3. Survival probabilities $S^{(N)}(t)$ obtained at $\eta=2$ for $\alpha=$ 0 (solid black lines) and $\alpha=2$ (solid blue lines) for up to $N=$ 5. (a) The green dots and red crosses mark the results obtained from Eqs. (19) and (20), respectively. (b) The dashed red lines show the results obtained from Eq. (18). The time and strength of the barriers are in $m L^{2} / \hbar$ and $\hbar^{2} /(m L)$, respectively.

changes of $S^{(N)}(t)$ in a short-time regime, and they were obtained by direct numerical integration in Eq. (10).

We can identify several distinct regions with different behaviors of $S^{(N)}(t)$ that deserve a profound investigation. To this end, we use a single-term approximation extracted from the resonance expansion of $S^{(N)}(t)$ in the following form:

$$
S^{(N)}(t) \approx \mathcal{N}_{n} e^{-\Gamma^{(n)} t} t^{-\beta},
$$

with

$$
\Gamma^{(n)}=\sum_{k=1}^{n} \Gamma_{k}, n \leq N,
$$

and the smallest exponent $\beta$, which we denote by $\beta(n, N)$. A close inspection reveals that $\beta(n, N)$ is in a simple relation with an exponent in the long-time asymptotic law for the TG system [16]:

$$
S^{(N)}(t) \propto t^{-\gamma(N)}
$$


$\gamma(N)=N(2 N+1)$, namely, $\beta(n, N)=\gamma(N-n)$. In this context, see also the informative discussion in Ref. [16]. Equation (15) can be expected to hold in cases when the true $S^{(N)}(t)$ resembles an exponential decay. For the reasons mentioned below Eq. (10), the formula in Eq. (15) also holds for the $N$-particle nonescape probability (only the factors $\mathcal{N}_{n}$ are different). However, it is important to stress that, in contrast to Eq. (17), Eq. (15) must be regarded as approximate.

Let us begin with the approximation for $n=N$ :

$$
S^{(N)}(t) \approx \mathcal{N}_{N} e^{-\Gamma^{(N)} t}
$$

Equation (18) has already appeared before in Ref. [7] and is valid to some extent, as confirmed by the results of Fig. 3(b). Although, at a short period, the $S^{(N)}(t)$ are only slightly dependent on $\alpha$, the effect of particle number is noticeable. To understand this phenomenon, we refer again to Fig. 1(c). Its inspection reveals that the sum of the two lowest decay rates $\Gamma_{r}$, that is, $\Gamma^{(2)}$, does not change much as $\alpha$ is varied. The presence of the additional particle prevents this effect, which manifests itself in the appearance of a stronger dependence of $\Gamma^{(3)}$ on $\alpha$. The same mechanism is responsible for the behaviors of $S^{(N)}(t)$ for a larger particle number $N$ as the results of Fig. 3(b) clearly indicate. It follows from the above that Eq. (15) is much more sensitive to changes in $\alpha$ for odd values of $n$ than for the even ones.

Next, we restrict ourselves to two particular time regions (long-lived) appearing before the long-time asymptotic regime. It can be expected that, in these regions, the $S^{(N)}(t)$ behave in accordance with Eq. (15) as follows:

$$
S^{(N)}(t) \approx \mathcal{N}_{1} e^{-\Gamma^{(1)} t} t^{-(2 N-3) N-1},
$$

and

$$
S^{(N)}(t) \approx \mathcal{N}_{2} e^{-\Gamma^{(2)} t} t^{-(2 N-7) N-6} .
$$

The first equation corresponds to the regime before to the transition to the long-time $t^{-\gamma(N)}$ regime. Note that for $N=2$, Eq. (20) reduces to Eq. (18). The results obtained from the above approximations are plotted along with the exact results in Fig. 3(a). For the clarity of the presentation, we display the results of Eq. (20) for only the case of $\alpha=2$ (we recall that $\Gamma^{(2)}$ is practically insensitive to changes in $\alpha$ ). Our results show an excellent agreement between the predictions of the above approximations and the exact results, thereby confirming the validity of Eq. (15) and disclosing the nature of the decay process of TG gases. It results from our study that only the initial phase of the decay of TG gases may follow an exponential law. The effect of the split barrier is most visible before the long-time asymptotic regime, which corresponds to Eq. (19). As far as we know, in none of the publications has Eq. (15) appeared.

Finally, we analyze the fraction of particles within the trap, which is now easily accessible by experimental ob-
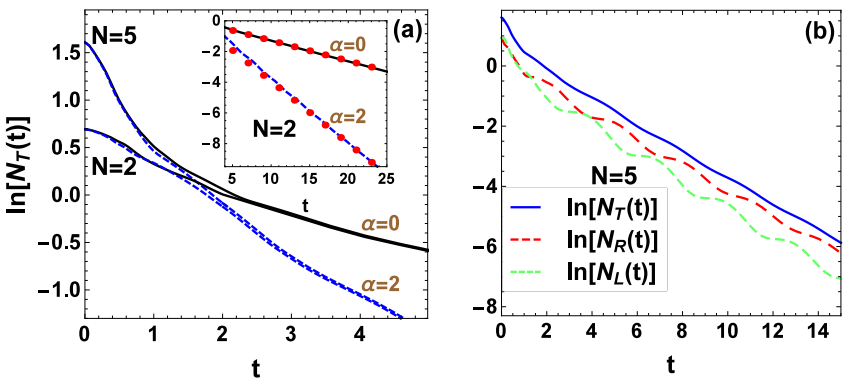

FIG. 4. (a) Results for the fraction of particles in the trap $N_{T}(t)$ obtained for the systems of $N=2$ and $N=5$ at $\eta=2$ for $\alpha=0$ (solid black lines) and $\alpha=2$ (dashed blue lines). The inset compares the results obtained from the resonance expansion approach (red dots, Eq. (22)) with the numerical ones. (b) Corresponding results obtained for $N_{L / R}(t)$ at $N=5$ and $\alpha=2$. The time and strength of the barriers are in $m L^{2} / \hbar$ and $\hbar^{2} /(m L)$, respectively.

servation techniques [22]:

$$
N_{T}(t)=\int_{-1}^{1} \rho(x, t) \mathrm{d} x
$$

where $\rho(x, t)$ is the one-body density matrix normalized to the number of particles $N$. Similarly, the fraction of particles in the left and right wells can be defined as $N_{L}(t)=\int_{-1}^{0} \rho(x, t) \mathrm{d} x$ and $N_{R}(t)=\int_{0}^{1} \rho(x, t) \mathrm{d} x$, respectively.

For the TG systems, Eq. (21) simplifies to the form $N_{T}(t)=\sum_{i=1}^{N} \rho_{i}(t)$, where $\rho_{i}(t)=\int_{-1}^{1}\left|\phi_{i}(x, t)\right|^{2} \mathrm{~d} x$. The time evolution of $N_{T}(t)$ was studied as a function of $N$ in Ref. [15] in the absence of a split barrier. Therefore, we only focus on the effect of the split barrier, limiting ourselves to systems with $N=2$ and $N=5$. Figure 4 (a) shows our numerical results for $N_{T}(t)$ calculated for the same control parameter values of the trap as those in Fig. 3. Figure 4(b) displays the corresponding results for $N_{L / R}(t)$ obtained for $N=5$ at $\alpha=2$. The time evolution of $N_{T}(t)$ can easily be understood from the facts established for the decay of one-particle states. At the beginning of the time period, the behaviors of $\rho_{i}(t)$ can be identified as consistent with the approximation: $\rho_{i}(t) \approx e^{-\Gamma_{i} t}$. Then, the quantity $N_{T}(t)$ is almost insensitive to changes in $\alpha$, as shown in Fig. 4(a). By contrast, $N_{T}(t)$ experiences an exponential decay when its components $\rho_{i}(t)$ go into the behaviors $\rho_{i}(t) \approx n_{i} e^{-\Gamma_{1} t}$, i.e.,

$$
N_{T}(t) \approx \mathcal{N}_{t} e^{-\Gamma_{1} t}
$$

where $\mathcal{N}_{t}=\sum_{i=1}^{N} n_{i}$. The validity of this approximation is verified both with and without the split barrier in the inset of Fig.4(a), where the values of $n_{i}$ were determined with the resonance expansion approach. We conclude that the presence of the split barrier primarily has a major impact on the exponential decay of $N_{T}(t)$. However, contrary to the behaviors of $N_{T}(t)$, those of $N_{L / R}(t)$ cannot be regarded as exponential. The slopes 
of $\ln N_{L / R}(t)$ display evident oscillations, which reflects the fact that resonance terms other than $e^{-\Gamma_{1} t}$ contribute considerably to $N_{L / R}(t)$. This phenomenon can be reasonably attributed to the effects of the particle tunneling between the wells.

\section{CONCLUSION}

In conclusion, we have carried out a detailed study of the decay properties of TG gases escaping from a double well modeled by Winter's system with the center $\delta$ split barrier. We provided an explicit representation of the time-dependent single-particle states in terms of the Fourier integrals, enabling accurate evolutions of the TG wave functions with time. Using the resonance expansion approach, we derived the closed-form approximate expression for the survival probability in the general case of $N$ particles. We demonstrated its validity at different stages of the time evolution, thus revealing the mechanism of the decay process of unstable TG gases. We also explained the parity effect appearing in the behavior of the survival probability in the presence of a split barrier. Our study establishes that only in the initial period can the decay of TG gases be regarded as purely exponential. In addition, we investigated the effect of the split barrier on the fraction of particles in the trap during the time evolution.

We leave open the question of how the features revealed in this study are affected if one considers the finite interaction strength and/or models with other confining potentials.The decay of many-particle systems exhibits an exponential behavior for any interaction strength (e.q., Ref. [26]); however, little is known about its course at further stages of the time evolution. In the finite interaction case, the classical counterpart of the system can have a dynamical instability. As a result, one can expect significant deviations from the decay features established in this paper for TG gases $[53,54]$. Another route for further study is to investigate in detail the effect of particle tunneling between the left and right wells.

We believe that our study will broaden discussion about the many-particle decay process.

\section{APPENDIX}

\section{A. Continuous spectrum eigenfunctions}

To find the time dependence of $\phi_{k}(x, t)$ we solved the Schrödinger equation (2) for the eigenstates of the continuous spectrum. The problem is equivalent to a free particle provided that its wave function is continuous in the whole space and satisfies specific matching conditions at the positions of the $\delta$ barriers [51]:

$$
\begin{gathered}
\lim _{\epsilon \rightarrow 0+}\left[\psi^{\prime}(\epsilon)-\psi^{\prime}(-\epsilon)\right]=2 \alpha \psi(0), \\
\lim _{\epsilon \rightarrow 0^{+}}\left[\psi^{\prime}(1+\epsilon)-\psi^{\prime}(1-\epsilon)\right]=2 \eta \psi(1) .
\end{gathered}
$$

We omit here the derivation details and report our final step:

$$
\psi_{p}(x)=\mathrm{A}(p)\left\{\begin{array}{l}
\sin (p x)+\tan (p) \cos (p x),-1 \leq x \leq 0 \\
\mathrm{~B}_{p} \sin (p x)+\tan (p) \cos (p x), 0<x<1 \\
\mathrm{C}_{p} \sin (p x)+\mathrm{D}_{p} \tan (p) \cos (p x), x \geq 1
\end{array}\right.
$$

where

$$
\begin{gathered}
\mathrm{B}_{p}=\frac{2 \alpha \tan (p)}{p}+1 \\
\mathrm{C}_{p}=2 \frac{\alpha \eta[1-\cos (2 p)]+\alpha p \tan (p)+\eta p \sin (2 p)}{p^{2}}+1 \\
\mathrm{D}_{p}=2 \frac{\alpha \eta[\cos (2 p)-1]-\eta p \sin (2 p)}{p^{2}}+1
\end{gathered}
$$

with $\mathrm{A}(p)$ determined by a standard normalization for the continuous spectrum,

$$
\int_{-1}^{\infty} \psi_{p^{\prime}}(x) \psi_{p}(x) \mathrm{d} x=\delta\left(p^{\prime}-p\right)
$$

that is,

$$
\mathrm{A}(p)=\frac{\sqrt{\frac{2}{\pi}} p^{2}}{\sqrt{4 \tan (p)[\alpha \tan (p)+p]\left\{\alpha\left(2 \eta^{2}+p^{2}\right)+2 \eta\left[\cos (2 p)\left(p^{2}-\alpha \eta\right)+p(\alpha+\eta) \sin (2 p)\right]\right\}+p^{4} / \cos ^{2}(p)}} .
$$

where $p=(2 E)^{1 / 2}$.

\section{B. Eigenfunctions of a hard- wall split trap}

Even-parity $(+)$ one-particle eigenfunctions of the hard-wall split trap are found as [52]

$\phi_{k}{ }^{(+)}(x, 0)=\mathrm{A}_{k}\left\{\begin{array}{l}\cos \left(p_{k} x\right)-\frac{\alpha}{p_{k}} \sin \left(p_{k} x\right),-1 \leq x \leq 0 \\ \cos \left(p_{k} x\right)+\frac{\alpha}{p_{k}} \sin \left(p_{k} x\right), 0<x \leq 1\end{array}\right.$ 
where $A_{k}$ is the normalization factor and the values of $p_{k}$ are the solutions of the transcendental equation: $\tan (p)=-p / \alpha$. The energies are given by $E_{k}^{(+)}=p_{k}^{2} / 2$ and ordered so as to form an increasing sequence. In the odd-parity case $(-)$ the wave functions vanish at $x=0$ and therefore are the same as those for the pure hard- wall trap; that is,

$$
\phi_{k}^{(-)}(x, 0)=\sin (\pi k x)
$$

with energies $E_{k}^{(-)}=(\pi k)^{2} / 2$.

\section{Expansion coefficients $C_{k}(p)$}

In both Eqs. (29) and (30), the integral in Eq. (7) can be performed algebraically:

$$
C_{k}^{(+)}(p)=\frac{2 \mathrm{~A}_{k} \mathrm{~A}(p) \sin (p)\left\{p\left(\alpha^{2}+p_{k}^{2}\right) \sin \left(p_{k}\right)-\tan (p)\left[p_{k}\left(\alpha^{2}+p^{2}\right) \cos \left(p_{k}\right)+\alpha\left(p^{2}-p_{k}^{2}\right) \sin \left(p_{k}\right)\right]\right\}}{\alpha p\left(p^{2}-p_{k}^{2}\right)}
$$

and

$$
C_{k}^{(-)}(p)=\frac{2 \mathrm{~A}(p) \pi k(-1)^{k} \sin (p)(\alpha \tan (p)+p)}{p\left(p^{2}-\pi^{2} k^{2}\right)}
$$

respectively. The coefficients $C_{k}(p)$ and the related quantities are ordered according to the energy spectrum of the split hard-wall trap as $C_{k}(p)=C_{k / 2}^{(-)}(p)$ and $C_{k}(p)=$

$$
C_{k / 2+1 / 2}^{(+)}(p) \text { for even and odd values of } k \text {, respectively. }
$$

\section{ACKNOWLEDGMENTS}

The author is thankful to Tomasz Sowiński and Arkadiusz Kuroś for their comments and suggestions on a draft of this article.

[1] G. Gamow, Z. Phys. 51, 204 (1928)

[2] E. U. Condon, R. W. Gurney, Nature (London) 122, 439 (1928)

[3] R. W. Gurney, E. U. Condon, Phys. Rev. 33, 127 (1929)

[4] G. Breit, E. P. Wigner, Phys. Rev. 49, 519 (1936)

[5] T. Jittoh, S. Matsumoto, J. Sato, Y. Sato, and K. Takeda, Phys. Rev. A 71, 012109 (2005)

[6] T. Koide, F. M. Toyama, Phys. Rev. A 66, 064102 (2002)

[7] M. Pons, D. Sokolovski, A. del Campo, Phys. Rev. A 85, 022107 (2012)

[8] G. Garcia-Calderón, R. Romo, Phys. Rev. A 93, 022118 (2016)

[9] K. Urbanowski, Eur. Phys. J. D 71, 118 (2017)

[10] G. Garcia-Calderón, J. L. Mateos, M. Moshinsky, Phys. Rev. Lett. 74, 337 (1995)

[11] R. G. Winter, Phys. Rev. 123, 1503 (1961)

[12] D. A. Dicus, W. W. Repko, R. F. Schwitters, and T. M. Tinsley, Phys. Rev. A 65, 032116 (2002)

[13] M. Peshkin, A. Volya, V. Zelevinsky, Europhys. Lett. 107, 40001 (2014)

[14] A. N. Petridis L. P. Staunton, J. Vermedahl, M. Luban, Modern Physics 1, 124 (2010)

[15] A. del Campo, F. Delgado, G. Garcia-Calderón, J. G. Muga, and M. G. Raizen, Phys. Rev. A 74, 013605 (2006)

[16] A. del Campo, Phys. Rev. A 84, 012113 (2011)

[17] A. del Campo, J. G. Muga, Europhys. Lett. 74, 965 (2006)

[18] S. R. Wilkinson, C. F. Bharucha, M. C. Fischer, K. W. Madison, P. R. Morrow, Q. Niu, B. Sundaram, and M. G. Raizen, Nature 387, 575 (1997)

[19] N. G. Kelkar, M. Nowakowski, K. P. Khemchandani, Phys. Rev. C, 70, 24601 (2004)

[20] A. Crespi, F. V. Pepe, P. Facchi, F. Sciarrino, P. Mataloni, H. Nakazato, S. Pascazio, and R. Osellame, Phys. Rev. Lett. 122, 130401 (2019)

[21] G. Zürn, F. Serwane, T. Lompe, A. N. Wenz, M. G. Ries, J. E. Bohn, and S. Jochim, Phys. Rev. Lett. 108, 075303 (2012)

[22] F. Serwane, G. Zürn, T. Lompe, T. B. Ottenstein, A. N. Wenz, and S. Jochim, Science 332, 6027 (2011)

[23] G. Garcia-Calderón, L. G. Mendoza-Luna, Phys. Rev. A 84, 032106 (2011)

[24] J. Dobrzyniecki, T. Sowiński, Phys. Rev. A 98, 013634 (2018)

[25] J. Dobrzyniecki, T. Sowiński, Phys. Rev. A 99, 063608 (2019)

[26] A. U. J. Lode, A. I. Streltsov, O. E. Alon, H.-D. Meyer, and L. S. Cederbaum, J. Phys. B 42, 044018 (2009)

[27] A. U. J. Lode, A. I. Streltsov, K. Sakmann, O. E. Alon, and L. S. Cederbaum, Proc. Natl. Acad. Sci. USA 109, 13521 (2012)

[28] S. E. Gharashi, D. Blume, Phys. Rev. A 92, 033629 (2015)

[29] S. Kim, J. Brand, J. Phys. B 44, 195301 (2011)

[30] I. S. Ishmukhamedov, A. S. Ishmukhamedov, Phys. E 109, 24 (2019)

[31] S. Hunn, K. Zimmermann, M. Hiller, and A. Buchleitner, Phys. Rev. A 87, 043626 (2013)

[32] T. Taniguchi, S. Sawada, Phys. Rev. E 83, 026208 (2011) 
[33] T. Maruyama, T. Oishi, K. Hagino, and H. Sagawa, Phys. Rev. C 86, 044301 (2012)

[34] N. Moiseyev, Physics Report 302, 211 (1998)

[35] A. Kuroś, A. Okopińska, Int. J. Theor. Phys., 54, 4164 (2015)

[36] P. Kościk, Phys. Lett. A 380, 1256 (2016)

[37] A. Kuroś, P. Kościk, A. Okopińska, J. Phys. A: Math. Theor. 46, 085303 (2013)

[38] A. Kuroś, A. Okopińska, Few-Body Systems 56, 853 (2015)

[39] M. D. Girardeau, J. Math. Phys. 1, 516 (1960)

[40] M. D. Girardeau, E. M. Wright, Phys. Rev. Lett. 84, 5691 (2000)

[41] S. Zöllner, H.-D. Meyer, P. Schmelcher, Phys. Rev. Lett. 100, 040401 (2008)

[42] S. Zöllner, H.-D. Meyer, P. Schmelcher, Phys. Rev. A 78, 013621 (2008)

[43] B. Chatterjee, I. Brouzos, S. Zöllner, and P. Schmelcher, Phys. Rev. A 82, 043619 (2010)
[44] B. Zhou, J-Q. Liang, F-C. Pu, Phys. Lett. A 281, 105 (2001)

[45] J. Dobrzyniecki, T. Sowiński, Phys. Lett. A 382, 394 (2018)

[46] J. Dobrzyniecki, T. Sowiński, Eur. Phys. J. D 70, 83 (2016)

[47] I. S. Ishmukhamedov, V. S. Melezhik, Phys. Rev. A 95, 062701 (2017)

[48] Y. Liu, Y. Zhang, Phys. Rev. A 91, 053610 (2015)

[49] M. Olshanii, Phys. Rev. Lett. 81, 938 (1998)

[50] S. Cordero, G. Garcia-Calderón, Phys. Rev. A 86, 062116 (2012)

[51] E. H. Lieb, W. Liniger, Phys. Rev., 1301605 (1963)

[52] X. Yin, Y. Hao, S. Chen, and Y. Zhang, Phys. Rev. A 78, 013604 (2008)

[53] W. Bauer, G. F. Bertsch, Phys. Rev. Lett. 65, 2213 (1990)

[54] O. Legrand, D. Sornette, Phys. Rev. Lett. 66, 2172 (1991) 